\title{
The breakdown of the Simon effect in cross-modal contexts: EEG
}

\section{evidence}

\author{
Leonor Castro $^{1}$, Salvador Soto-Faraco ${ }^{1,2}$, Luis Morís Fernández ${ }^{1}$, Manuela \\ Ruzzoli $^{1}$ \\ ${ }^{1}$ Center for Brain and Cognition, Departament de Tecnologies de la Informació i les \\ Comunicacions, Universitat Pompeu Fabra, 08018 Barcelona, Spain \\ ${ }^{2}$ Institució Catalana de Recerca i Estudis Avançats (ICREA)
}

\section{Address:}

Center for Brain and Cognition, Universitat Pompeu Fabra, Edifici Mercè Rodoreda, carrer Ramon Trias Fargas, 25-27, 08005 Barcelona, Spain.

\section{Authors' contribution:}

L.C. designed the experiments, collected and analyzed the data, interpreted the results, wrote the manuscript.

S.S.F. designed the experiments, interpreted the results, and wrote the manuscript. L.M. analyzed the data, interpreted the results, and wrote the manuscript. M.R. designed the experiments, planned and realized data analyses, interpreted the results, wrote the manuscript.

\section{Correspondence should be addressed to}

M. R. (manuela.ruzzoli@gmail.com) Center for Brain and Cognition, Universitat Pompeu Fabra, Edifici Mercè Rodoreda, carrer Ramon Trias Fargas, 25-27, 08005 Barcelona, Spain.

Running Title: EEG evidence in cross-modal Simon effect

Keywords: Lateralized Readiness Potential (LRP), P300, Stimulus-Response compatibility, Theta oscillations 


\begin{abstract}
In everyday life, we often must coordinate information across spatial locations and different senses for action. It is well known, for example, that reactions are faster when an imperative stimulus and its required response are congruent than when they are not, even if stimulus location itself is completely irrelevant for the task (the so-called Simon effect). However, because these effects have been frequently investigated in single modality scenarios, the consequences of spatial congruence when more than one sensory modality is at play are less well known. Interestingly, at a behavioural level, the visual Simon effect vanishes in mixed (visual and tactile) modality scenarios, suggesting that irrelevant spatial information ceases to exert influence on vision. In order to shed some light on this surprising result, here we address the expression of irrelevant spatial information in EEG markers typical of the visual Simon effect (P300, theta power modulation, LRP) in mixed modality contexts. Our results show no evidence for the visual spatial information to affect performance at behavioural and neurophysiological levels. The absence of evidence of the neural markers of visual S-R conflict in the mixed modality scenario implies that some aspects of spatial representations that are strongly expressed in single modality scenarios might be bypassed.
\end{abstract}




\section{Introduction}

A large body of empirical evidence demonstrates that the location of an event often has a strong influence on the observer's reactions to it, even when stimulus location itself bears no relevance. This is a well-established principle in design (for a recent review of human-computer interfaces see Proctor \& Vu, 2016), to optimize human performance and prevent potentially dangerous mistakes, a required action (i.e., the button a user must operate upon) should ideally be spatially compatible with the location where the imperative information prompting the action appears. The Simon task, a classical example of this kind of spatial stimulus-response (S-R) compatibility, requires participants to press different buttons according to the color of a stimulus (i.e., a nonspatial stimulus dimension) that can appear either to the right or to the left side of fixation. The Simon effect reflects in faster and more accurate responses when the location of the stimulus matches the location of the required response (i.e., compatible trials), compared to when their positions mismatch (i.e., incompatible trials) (Simon \& Small, 1969).

The Simon effect is a fairly robust phenomenon that occurs with visual (Wallace, 1971; Riggio et al., 1986), auditory (Simon et al., 1970; Roswarski \& Proctor, 1996; Röder et al., 2007), and tactile (Medina et al., 2014; Ruzzoli \& Soto-Faraco, 2017) stimuli, thus providing a reliable protocol for testing how task irrelevant stimulus features (i.e., space) affect responses in different sensory modalities and, more generally, how spatial information is represented in the brain (Proctor \& Lu, 1999; Hommel, 2011). Accordingly, it has been found that the reference frame in which spatial information is represented is strongly dependent of stimulus modality (Wallace, 1971; Riggio et al., 1986; Hommel, 1993; Medina et al., 2014; Wang et al., 2016; Ruzzoli \& Soto-Faraco, 2017): in vision and in audition, S-R compatibility abides to an external reference 
frame, so that the relevant matching is between the position of the stimulus and the position of the response goal (Wallace, 1971; Riggio et al., 1986). Conversely, for touch, S-R compatibility abides to an anatomical reference frame so that what matters is the compatibility between the position of the stimulus on the body and the anatomical side of the hand used to respond (Medina et al., 2014; Ruzzoli \& Soto-Faraco, 2017). A commonly accepted view is that multiple spatial codes, derived from stimulus location, body posture and response goal are automatically encoded (Proctor \& Lu, 1999; Hommel, 2011) and interact in action planning (Wallace, 1971; Hommel, 1993). However, this framework has been recently challenged by Ruzzoli \& Soto-Faraco (2017) based on the consequences of mixing events in different modalities in a Simon task. Mixing visual and tactile events (coded in external and anatomical reference frames, respectively) in an unpredictable fashion led the Simon effect to vanish in vision (but to remain for touch). Instead, modality mixing between visual and auditory stimuli (which partake S-R compatibility patterns in the external space) resulted in a preserved Simon effect for both modalities. Therefore, in situations in which multiple reference frames can be formed, some instances of spatial S-R compatibility may disappear (Ladavas \& Moscovitch, 1984; Umiltà \& Liotti, 1987; Ruzzoli \& SotoFaraco, 2017), thus suggesting that the impact of spatial information (compatible vs. incompatible) is less automatic than initially assumed, and modulated by contextual factors (Pouget et al., 2002; Heed et al., 2015). An automatic coding of irrelevant spatial information (Proctor \& Lu, 1999) would have led the Simon effect to be present even in the modality switching context. This is remarkable, because in everyday life situations it is often the case that events in various modalities convey spatial information. The consequence of S-R compatibility in multiple-modality scenarios is therefore a relevant, yet fairly unexplored, issue. One standing question is whether the 
processing of visual spatial information has an impact in the neural markers of stimulus processing in multisensory scenarios despite the lack of Simon effect on RTs.

Answering this question is important because it can shed light on the underlying mechanisms and chain of events responsible for S-R compatibility effects in everyday life contexts.

Here, we measured the expression of known neural EEG markers of visual S-R compatibility, both in mixed modality and in single modality scenarios. If these neural markers are expressed in a similar way in the single and mixed modality context, despite differences at behavioural level, then one would accept currently dominant accounts (Lu \& Proctor, 1995; Hommel, 2011) suggesting that spatial information is indeed automatically encoded (although its behavioral expression is not manifested in multisensory contexts). Alternatively, the absence of the neural markers of S-R conflict in mixed modality contexts (or a reduction in comparison to the single modality context) would imply that the encoding of different sources of spatial information (coming from the stimulus, the response, and the responding agent) and its ensuing impact on behavior can be modulated by contextual factors (Pouget et al., 2002; Heed et al., 2015).

We focused on three well known EEG indexes which have been widely investigated in the Simon task: the P300 peak latency shift, the increase in power of the oscillatory activity in the theta band $(5-7 \mathrm{~Hz})$, and the positive dip in the Lateralized Readiness Potential (LRP). According to previous literature on each of these markers, the P300latency shift is a measure of perceptual interference (Ragot \& Renault, 1981; ValleInclán, 1996), the increase in EEG theta power indicates executive function interference (Cohen, 2014), and LRP modulation indicates motor response interference (ValleInclán, 1996). We measured these neural markers as participants were engaged in a 
mixed modality (visuo-tactile) Simon task in which we expected to observe a behavioral attenuation (or complete disappearance) of the visual Simon effect and preserved tactile Simon effect (Ruzzoli \& Soto-Faraco, 2017). In order to interpret negative findings in the mixed-modality scenario (expected for the visual modality), we introduced single modality Simon blocks (either visual or tactile) for which we predicted the presence of the Simon effect in terms of RTs and the consequent EEG markers, according to extant literature. The focus of interest in this study was the visual modality. First, because vision is the most altered modality in the mixed modality situations and, second, because past EEG literature in the Simon task has mostly concentrated on the visual modality, so we can set precise expectations about its neural markers. We outline below the expected findings regarding the EEG indexes of the Simon effect and the main hypotheses in a mixed modality context.

The P300 is a positive ERP component, elicited about 250-350 ms after stimulus onset and most visible at centroparietal electrodes. It has been shown that trial to trial variations in P300 latency correlate with RTs (Ritter et al., 1972). In the Simon task, at least for the visual modality, P300 latency is delayed in incompatible trials compared to compatible trials (Ragot \& Renault, 1981; Valle-Inclán, 1996; Leuthold \& Sommer, 1999; Masaki et al., 2000; Ideno et al., 2005; Galashan et al., 2008; Melara et al., 2008). This P300-latency shift in incompatible trials has been interpreted as a marker of perceptual interference, stressing the idea that extracting the relevant stimulus attribute (i.e., color) might take longer in incompatible than compatible trials (hence, perceptual interference; Hasbroucq \& Guiard, 1991; Leuthold, 2011; Ragot \& Renault, 1981; Valle-Inclán, 1996). Based on previous evidence, we can envision two different scenarios in the mixed modality Simon task: if the P300 latency for visual trials is not modulated (not delayed in incompatible trials), we should conclude that, similarly to 
RTs, the perceptual interference ensuing a stimulus-response-incompatibility is negligible. Alternatively, if the visual P300 latency modulation is present, despite of the lack of behavioral Simon effect, then we should conclude that S-R compatibility does in fact delay perceptual analysis of the relevant attribute when a more sensitive index is taken into account (i.e., P300), in contrast to RTs. This would mean that the irrelevant spatial information in vision does not cease to generate S-R compatibility effects on perception in mixed modality contexts, but its impact is attenuated and only evident from ERPs.

Another EEG correlate of the Simon effect is an increase in the power of oscillatory activity in the theta band $(5-7 \mathrm{~Hz})$ over the medial frontal cortex in incompatible trials in comparison to compatible trials (Nigbur et al., 2011; Cohen \& Ridderinkhof, 2013; Cohen, 2014). This neural correlate is considered a marker of cognitive interference, typically observed in the Simon task (Nigbur et al., 2011; Cohen \& Donner, 2013; Gulbinaite et al., 2014; Töllner et al., 2017), as well as in other conflict tasks such as the Stroop (Stroop, 1935) and the Eriksen flanker task (Eriksen \& Eriksen, 1974). Hence, the stronger post-stimulus theta power in incompatible trials is the result of a competition (i.e., conflict) between the response automatically primed by stimulus location and the task-relevant response that is actually required (Cohen, 2014). In our experiment, the presence of an increased theta power in incompatible visual trials in the mixed modality Simon task would reflect the presence of conflict, even if stimulus location fails to affect RTs at a behavioral level. In this case, one would think that irrelevant spatial information can generate cognitive conflict at the level of executive control, but under a mixed modality context, this conflict is resolved as efficiently in compatible as in incompatible trials, hence not leading to the behavioral Simon effect. 
Another ERP correlate in the Simon task is the Lateralized Readiness Potential (LRP), which provides a measure of covert motor activation for lateralized responses (Coles, 1989). The LRP provides information complementary to the perceptual or executive function interference, discussed so far. Classically, in the Simon task, incompatible trials induce an early motor activation of the incorrect hand (which is the spatially congruent hand). This expresses as a positive peak in the LRP, also called LRP-dip (Vallesi et al., 2005), right before the LRP deflection of the correct hand activation (negative deflection). In addition to the LRP dip, the typical LRP of the correct hand activation does appear in incompatible trials but later than in compatible ones (ValleInclán, 1996; Vallesi et al., 2005; Cespón et al., 2012). The LRP-dip in incompatible trials reflects automatic response activation triggered by the location of the stimulus, thus representing the influence of irrelevant spatial information on response selection (Gratton et al., 1988; Vallesi et al., 2005). This makes the LRP a useful marker of response-selection processes (Lu \& Proctor, 1995) in the Simon task and, consequently, relevant for the mixed modality scenario studied here. Specifically, if the LRP-dip would be attenuated or absent for the visual incompatible trials in the mixed modality scenario we should conclude that the irrelevant spatial information does not induce motor interference, in line with the RTs evidence. The alternative hypothesis is that, the LRP-dip would be visible and similar to the single modality Simon task, thus suggesting automatic motor priming of the corresponding (but irrelevant) stimulus location, contrary to the evidence coming from the RTs.

Please note that although previous literature has put the stress on different cognitive stages of the S-R interference for each of these neural markers (Valle-Inclán, 1996), it is best to see these neural correlates as not independent, possibly having overlapping causal origins and therefore informing on complementary aspects of the same process. 
That is, if there is no motor activation of the irrelevant response hand (i.e., LRP), there might not be a chance for cognitive conflict (i.e., theta power modulation). Furthermore, the P300 and the theta power modulation might be inter-independent signals (Başar et al., 1992, 1999; but see Cohen \& Donner, 2013), although it has been suggested that the two markers index different functional roles (perceptual evaluation and cognitive conflict, for the P300 and theta power modulation, respectively) (Cohen \& Donner, 2013). Yet, it is important to clarify that we do not assume complete independence between these markers, nor a particular temporal sequence in the relevant underlying mental processes that generate them.

\section{Materials and Methods}

\section{Participants}

Data from 22 healthy participants (with normal or corrected-to-normal vision) were recorded. After EEG artifact rejection, participants left with less than $50 \%$ of the total number of trials were excluded, leaving a total of 15 participants ( 2 left-handed; 8 female; age-range between 18 and 38 years) for the final analysis. The experiment was run in accord to the Declaration of Helsinki and approved by the ethics committee CEIC Parc de Mar (University Pompeu Fabra, Barcelona, Spain). Participants gave written informed consent before participating in the study and received a compensation of 10 euro/hour for their time.

\section{Apparatus and stimuli}

The experiment was designed and executed using Psychtoolbox (Brainard, 1997) on MatLab R2010b. The stimuli and the procedure were in close replication of Ruzzoli \& Soto-Faraco (2017).

Visual stimuli: Visual stimuli were presented through a CRT monitor (1024 x 768 pixels), with refresh rate of $100 \mathrm{~Hz}$ and true color (32bit). The visual stimulus was a 
disk ( $\varnothing=4.2^{\circ}$ of visual angle, v.a. henceforth), presented $8.2^{\circ}$ of v.a. either right or left from a central fixation cross for $100 \mathrm{~ms}$. Stimulus color could be light (contrast=23 $\mathrm{cd} / \mathrm{m}^{2}$ ) or dark gray (contrast $=3 \mathrm{~cd} / \mathrm{m}^{2}$ ) presented on a uniform mid-gray background (contrast $\left.=8 \mathrm{~cd} / \mathrm{m}^{2}\right)$. Each combination of disk contrast (dark/light) and position (left/right) was equally probable, and unpredictable.

Tactile stimuli: Vibrotactile stimuli were delivered through Oticon-A bone-conduction vibrators $\left(3.8 \mathrm{~cm}^{2}\right.$ vibrating surface; Oticon) positioned just below the nail of the index finger, of the left and right hand. The vibrotactile stimulus lasted for $100 \mathrm{~ms}$ (10 ms ramps) and it was a sine wave vibration either of $100 \mathrm{~Hz}$ (weak stimulus) or of $200 \mathrm{~Hz}$ (strong stimulus). Each combination of stimulus strength (dark/light) and position (left/right hand) was equally probable, and unpredictable. To mask any possible noise produced by the vibrotactile stimulators, participants listened to white noise through in ear headphones during the entire duration of the experiment.

\section{EEG recording}

EEG was continuously recorded through 60 active electrodes (actiCAP, Brain Products $\mathrm{GmbH}$, Munich, Germany) placed after the 10-10 international system, with AFz as ground, and reference electrode placed on the tip of the nose. Horizontal and vertical electro-oculograms (hEOG and vEOG) were recorded from two additional electrodes placed at the outer canthus and under the right eye, respectively. Other two additional electrodes were placed over the left and right mastoids for off-line re-referencing. Impedance was kept below $10 \mathrm{k} \Omega$. The EEG signal was recorded via BrainVision Recorder (Brain Products GmbH, Munich, Germany) at a sampling rate of 500Hz.

\section{Procedure}

The experiment was performed in a dimly lit, sound attenuated room, and lasted about 2.5 hours, breaks included. Each participant completed four different blocks, in the 
following order. First, two single modality blocks (one visual and one tactile, the order was counterbalanced across participants) of 320 trials each, then one mixed modality block (trials were intermixed between visual and tactile modalities) of 416 trials, and finally a second single visual modality block (320 trials). We chose a "sandwich-like" paradigm in which the mixed modality Simon block was embedded between the two visual single modality blocks, in order to measure possible carry-over effects from the mixed modality task (from which we expected no visual Simon effect, based on Ruzzoli \& Soto-Faraco, 2017). Each block was preceded by training (16 trials in the single modality blocks, and 32 trials in the mixed modality block) in order to familiarize participants with the procedure and the response mapping. If participants explicitly reported uncertainty, the training session was repeated. Data from the training sessions were not stored.

Participants rested their hands, palm down, with the index fingers positioned on the response keys of the computer keyboard ('q' and 'p' for left and right hand responses, respectively). During the experiment, participant's arms and hands were covered from sight with a black cloth in order to block visual information related to body posture, which might affect tactile processing (Yamamoto \& Kitazawa, 2001), although participants were instructed to maintain the fixation on a black central cross $\left(0.6^{\circ}\right.$ of v.a.) presented on the screen for the entire duration of the trial. At the beginning of each trial, the fixation cross flashed in white three times $(100 \mathrm{~ms} / 100 \mathrm{~ms}$, on/off periods) followed, after a delay randomly chosen between 300 and $500 \mathrm{~ms}$, by the target stimulus (visual or tactile). Participants were instructed to respond as fast as possible to the color (visual Simon task) or to the vibrotactile frequency of the stimulus (tactile Simon task), depending on the modality of the event, and irrespective of its spatial position. Responses were provided by pressing the "q" or "p" key on a computer 
keyboard with the index finger of each hand. The next trial started after a randomly jittered 800-1000 ms window following response. The response mapping (response associated to each stimulus) was counterbalanced across participants, so that one half of the participants responded to the dark disk with the right hand, and strong tactile vibration with the right hand, and the other half of participants responded to the light disk with the left hand, and soft tactile vibration with the left hand. In all blocks (single or mixed modality), the response instructions were formulated stressing the mapping between the anatomical effector (right/left hand) and the corresponding stimulus feature (color/vibrotactile intensity).

\section{Data pre-processing}

RTs slower than $1500 \mathrm{~ms}$ and incorrect responses were excluded from behavioral and EEG analyses. The EEG signal was analyzed using BrainVision Analyzer 2 (Brain Products GmbH, Munich, Germany) for the P300 calculation, and Matlab (Release 2016b) and Fieldtrip (Oostenveld et al., 2011) for the LRP and theta power analysis. First, the raw EEG signal was re-referenced to the averaged mastoids, then band-pass filtered $(0.01-30 \mathrm{~Hz}$ for the P300 and LRP; $0.5-50 \mathrm{~Hz}$ for the theta power). The EEG was segmented in epochs from $-200 \mathrm{~ms}$ to $+1500 \mathrm{~ms}$, time locked to the target onset. Eye movements, blinks and muscle artifacts were detected in a semi-automatic way, by bipolar horizontal and vertical EOG derivations. The minimum voltage accepted for artifact rejection was individually adjusted so that less than $10 \%$ of the total amount of trials had to be rejected.

P300: After segmentation, DC-detrend was applied to data from one participant in the visual and tactile single modality blocks because of the presence of slow frequency drift in the recording. Baseline correction was applied considering as baseline $200 \mathrm{~ms}$ before stimulus onset. The P300 peak latency and amplitude was extracted semi-automatically 
from the average of each participant, as the largest positive voltage value within the 250-500 ms post-stimulus window at the centro-parietal cluster $\{\mathrm{Cz}, \mathrm{C} 3, \mathrm{C} 4, \mathrm{CPz}, \mathrm{CP} 3$, CP4\}.

Theta power: In order to extract theta power, we calculated the Short Time Fourier Transform (STFT) for each epoch (from $-200 \mathrm{~ms}$ to $+1500 \mathrm{~ms}$ stimulus locked) using a $500 \mathrm{~ms}$ Hanning window in $20 \mathrm{~ms}$ steps, in a spectral window from $2 \mathrm{~Hz}$ to $30 \mathrm{~Hz}$ in steps of $1 \mathrm{~Hz}$; the size of the Hanning window was optimized so it included 3 cycles of the central frequency $(6 \mathrm{~Hz})$ of the band of interest $(5-7 \mathrm{~Hz})$. The power (in $\mathrm{dB}$ ) of the signal resulting from the STFT was averaged across trials for each condition and block, separately for each participant. As the time period between the fixation and the stimulus presentation was jittered between $300-500 \mathrm{~ms}$, too short to create a proper baseline, we discarded baseline correction and focused our analysis on the difference between compatible and incompatible trials within each block.

We exclusively analyzed the theta power in the range $5-7 \mathrm{~Hz}$ at a fronto-medial electrodes cluster $\{\mathrm{Fz}, \mathrm{FCz}, \mathrm{Cz}\}$ (Cohen \& Donner, 2013; Cohen \& Ridderinkhof, 2013; Cohen, 2014). If one electrode in the cluster was contaminated by exceeding noise, the noisy electrode was excluded from the cluster (this happened in five participants: Fz in two participants and FCz in four participants). Theta-band power was inspected for peak detection in the time window between 150 and $550 \mathrm{~ms}$ post stimulus (Cohen \& Ridderinkhof, 2013) for each condition and modality block. We extracted the amplitude (80 ms-average around the peak) of each peak. For most of the participants a single peak in the theta power was found for each condition, however in some cases $(\sim 2 \%$ of the total) it was not possible to find a clear peak in the selected time window. When no clear peak was identified in a condition for a participant (one participant in visuo-tactile block and another participant in the second visual block) that data point 
from the participant was excluded for the statistical analysis, instead of entirely excluding the participant from the dataset (this results in different degrees of freedom in statistical analyses, depending on the specific contrasts).

LRP: One potential worry in the LRP analysis (especially in speeded tasks, like the Simon paradigm) is the contamination of motor potentials by sensory potentials due to the lateralized targets (Valle-Inclán, 1996; Eimer, 1998). A way to mitigate this possible confound is to use the Surface Laplacian (SL) transformation (Carvalhaes \& de Barros, 2015), a method that improves spatial resolution (SL estimates have a sharper topography, reducing the negative impact of volume conduction) (Kayser \& Tenke, 2015; Carvalhaes \& de Barros, 2015). We applied the SL transformation to each epoch following the spherical spline interpolation procedure described by Perrin et al. (1989), implemented in Fieldtrip (Oostenveld et al., 2011), (conductivity $=0.33 \mathrm{~S} / \mathrm{m}$, order of the spline $=4$, degree of the polynomial $=14$, regularization $=10^{-5}$ ). Data from one more participant was excluded, due to excessive noise in electrode C3. The LRP was then calculated for the remaining 14 participants from the SL transformed signal as in Coles (1989).

$$
\frac{[(C 3-C 4) \text { right hand }+(C 4-C 3) \text { left hand }]}{2}
$$

The right and left hand refer to the hand corresponding to the correct response, according to task instructions, and the $\mathrm{C} 3-\mathrm{C} 4$ and $\mathrm{C} 4-\mathrm{C} 3$ are the electrical potential difference between $\mathrm{C} 3$ and $\mathrm{C} 4$ electrodes, putatively localized above pre-motor and motor areas. According to this formula, negative polarity reflects the activation of the motor pathway leading to a correct response, whereas positive polarity reflects the activation of the alternative motor pathway.

\section{Statistical analyses}


Response mapping (which hand should be used for the correct responses according to task instructions) and order (order of the first two blocks within the experiment) were initially included in the analyses as between participants' factors. However, they did not reach significance (response mapping: $\mathrm{F}(3,7)=1.99, \mathrm{p}=0.203, \eta^{2}=0.46$; order: $\left.\mathrm{F}(1,7)=0.004, \mathrm{p}=0.950, \eta^{2}<0.01\right)$ nor interacted with other factors in any block; therefore, we did not consider them any further in the analyses. Behavioral data (RT and accuracy) from the first three blocks (visual and tactile single modality and mixed modality blocks) were submitted to a repeated measures ANOVA with Context (single/mixed modality), Modality (visual/tactile), and S-R Compatibility (compatible/incompatible) as within participants' factors. Statistical significance was set to $\alpha<0.05$. One-tailed paired-t tests were run for post-hoc comparisons to contrast the hypotheses $\mathrm{RT}_{\text {compatible }}<\mathrm{RT}_{\text {incompatible }}$ and $\mathrm{Accuracy}_{\text {incompatible }}<$ Accuracy $_{\text {compatible. This }}$ analysis specifically aimed at replicating the Simon effect in the single modality Simon blocks and confirming the previous behavioral finding that the visual Simon effect vanishes when visual and tactile trials are intermixed (Ruzzoli \& Soto-Faraco, 2017). For the EEG analyses (P300, theta power, and LRP) we first evaluated the expected difference between compatible and incompatible trials in the single modality Simon blocks through one-tailed paired t-tests. For P300 and LRP, similar to the behavioral analysis (for the first three blocks, i.e., visual and tactile single modality and mixed modality blocks), we conducted a repeated measures ANOVA with Context (single/mixed modality), Modality (visual/tactile), and S-R Compatibility (compatible/incompatible) as within participants' factors. This analysis should reveal modulations in the Simon effect (S-R compatibility) across blocks and modalities. For the theta power, since we did not apply baseline correction, we performed a repeated measures ANOVA with Context (single/mixed modality), and Modality (visual/tactile), 
as within participants' factors on the difference in the peak amplitude of the theta power $(\mathrm{dB})$ between incompatible and compatible trials within each block.

\section{Results}

\section{Behavioral}

The ANOVA results showed a main effect of Context, because single modality RTs were faster compared to mixed modality ones (467 vs. $562 \mathrm{~ms}$, respectively; $\left.F(1,14)=69.62, p<0.001, \eta^{2}=0.83\right)$. Overall, responses to visual stimuli were faster than responses to tactile stimuli (404 vs. $625 \mathrm{~ms}$, respectively), leading to a significant main effect of Modality $\left.\mathrm{F}(1,14)=216.86, \mathrm{p}<0.001, \eta^{2}=0.94\right)$. The main effect of $S-R$ Compatibility also reached significance, as compatible trials led to faster responses than incompatible trials (494 vs. $535 \mathrm{~ms}$, respectively; $\mathrm{F}(1,14)=23.67, \mathrm{p}<0.001, \eta^{2}=0.63$ ). Of interest for the purposes of this study, the interaction between Context, Modality and $S-R$ Compatibility was significant $\left[\mathrm{F}(1,14)=9.18, \mathrm{p}=0.009, \eta^{2}=0.40\right]$. As shown in Figure 1, the Simon effect was present in the single modality blocks both for the visual $\left(34 \mathrm{~ms} ; \mathrm{t}(14)=-6.55, \mathrm{p}<0.001 ; \mathrm{d}_{\mathrm{z}}=1.69\right)$ and tactile modalities $(45 \mathrm{~ms} ; \mathrm{t}(14)=-2.84$, $\mathrm{p}=0.013 ; \mathrm{d}_{\mathrm{z}}=0.73$ ), whereas in the mixed modality blocks, the visual Simon effect was strongly attenuated $(13 \mathrm{~ms})$ and did not reach significance $(\mathrm{t}(14)=-1.59, \mathrm{p}=0.135$; $\left.d_{z}=0.15\right)$, while it remained significant in the tactile modality $(73 \mathrm{~ms} ; \mathrm{t}(14)=-5.22$, $\left.\mathrm{p}<0.001 ; \mathrm{d}_{\mathrm{z}}=0.71\right)$. Finally, the visual Simon effect was present, again, in the second single modality block $\left(42 \mathrm{~ms} ; \mathrm{t}(14)=-5.95, \mathrm{p}<0.001 ; \mathrm{d}_{\mathrm{z}}=1.54\right)$, attesting the short lasting consequences of mixing modalities on the visual Simon effect. Please note that, in the mixed modality block, taking into account the modality of the previous trial (modality maintain vs. modality switch) did not alter the pattern of results (repeated measures ANOVA returned a non significant interaction modality trial $N \mathrm{x}$ modality transition $\mathrm{x}$ $S-R$ compatibility $\left.\mathrm{F}(1,14)=1.42, \mathrm{p}=0.247, \eta^{2}=0.10\right)$. Yet, the Simon effect in maintain 
visual trials was on average of $24 \mathrm{~ms}$, numerically higher than visual switch trials (preceded by a tactile) trial, where it was completely absent (-1 ms). Accuracy was overall high (91\%) and the results were in line with the RT results, yet less robust: Participants' responses were more accurate in the single modality than in mixed modality blocks ( $92 \%$ vs. $88 \%$, respectively; $\left.F(1,14)=43.89, p<0.001, \eta^{2}=0.76\right)$, and in the visual modality than in the tactile modality (94\% vs. $86 \%$, respectively; $\left.\mathrm{F}(1,14)=27.04, \mathrm{p}<0.001, \eta^{2}=0.66\right)$. The main effect of $S-R$ Compatibility was significant, because responses to compatible trials were more accurate than to incompatible trials (93\% vs. $87 \%$, respectively; $F(1,14)=28.29, \mathrm{p}<0.001, \eta^{2}=0.67$ ). The interaction between Context, Modality and $S$-R Compatibility was close to significance $\left[F(1,14)=4.17, p=0.060, \eta^{2}=0.23\right]$, reflecting a pattern consistent with the RTs.

Considering only visual modality trials, the Simon effect was completely absent in the mixed modality block (compatible $=93 \%$ vs. incompatible $=92 \%, \mathrm{t}(14)=0.74, \mathrm{p}=0.469$; $\left.d_{z}=0.19\right)$, but significant in the single modality blocks before (98\% vs. $93 \% ; t(14)=3.02$; $\left.\mathrm{p}=0.009 ; \mathrm{d}_{\mathrm{z}}=0.78\right)$ and after $\left(97 \%\right.$ vs. $\left.90 \%, \mathrm{t}(14)=4.01 ; \mathrm{p}=0.001 ; \mathrm{d}_{\mathrm{z}}=1.04\right)$ the mixed modality block.

The present behavioral pattern represents a close replication of the previous findings from Ruzzoli \& Soto-Faraco (2017). As expected, when visual and tactile trials are unpredictably intermixed in a Simon task, the visual Simon effect was strongly reduced, while the tactile Simon effect remained unaltered. One novel piece of information is that the effect of modality switching does not have long lasting consequences, as the visual Simon effect reappeared when a single modality block was run right after the mixed modality block. The tactile version of the Simon effect occurred regardless of the single or mixed modality nature of the context. 
Please note that, although RTs to tactile stimuli are overall slower than RTs to visual stimuli, and the Simon effect has been found to be stronger for fast RTs (De Jong et al., 1994), the tactile Simon effect remains in the mixed modality context. To further explore the possibility that the overall slow-down of responses in the mixed modality block led the Simon effect to disappear, we performed a RTs distribution analysis on visual trials. RTs to visual stimuli for each participant were considered separately according S-R compatibility (compatible, incompatible) and then distributed into quartiles based on RTs speed. We ran a repeated measure ANOVA with Block (first single modality block, mixed modality block and second single modality block), Quartile (Q1-Q4) and S-R compatibility (compatible/incompatible) as within subject variables. As of main interest here, the triple interaction was significant $[F(6,84)=2.38$, $\left.p=0.036, \eta^{2}=0.15\right]$. Specifically, in the single modality blocks, the Simon effect was present in all quartiles $\left(\mathrm{t}_{\text {quartile } 1}(14)=-10.57 ; \mathrm{p}<0.001 ; \mathrm{d}_{\mathrm{z}}=0.89 ; \mathrm{t}_{\text {quartile2 }}(14)=-12.31\right.$; $\mathrm{p}<0.001 ; \mathrm{d}_{\mathrm{z}}=0.72 ; \mathrm{t}_{\text {quartile3 }}(14)=-6.00 ; \mathrm{p}<0.001 ; \mathrm{d}_{\mathrm{z}}=0.43 ; \mathrm{t}_{\text {quartile } 4}(14)=-2.10 ; \mathrm{p}=0.054 ;$ $d_{z}=0.15$. Visual single modality second block: $t_{\text {quartile } 1}(14)=-10.86 ; p<0.001 ; d_{z}=0.91$; $\mathrm{t}_{\text {quartile } 2}(14)=-8.72 ; \mathrm{p}<0.001 ; \mathrm{d}_{\mathrm{z}}=0.76 ; \mathrm{t}_{\text {quartile } 3}(14)=-7.35 ; \mathrm{p}<0.001 ; \mathrm{d}_{\mathrm{z}}=0.55 ; \mathrm{t}_{\text {quartile4 }}(14)=-$ 2.35; $\left.\mathrm{p}=0.034 ; \mathrm{d}_{\mathrm{z}}=0.24\right)$. In contrast, in the mixed modality block the Simon effect was present only in the first quartile (faster RTs) $\left(\mathrm{t}_{\text {quartile1 }}(14)=-3.99 ; \mathrm{p}=0.001 ; \mathrm{d}_{\mathrm{z}}=0.81\right)$ and there was only a tendency in the second quartile $\left(\mathrm{t}_{\text {quartile } 2}(14)=-2.13 ; \mathrm{p}=0.052 ; \mathrm{d}_{\mathrm{z}}=0.23\right.$; $\left.\mathrm{t}_{\text {quartile3 }}(14)=0.60 ; \mathrm{p}=0.556 ; \mathrm{d}_{\mathrm{z}}=0.05 ; \mathrm{t}_{\text {quartile4 }}(14)=-0.56 ; \mathrm{p}=0.587 ; \mathrm{d}_{\mathrm{z}}=0.06\right)$. This pattern reflects the well-known effect of overall RT on the Simon effect (De Jong et al., 1994), but also the fact that the Simon effect vanished (or at best reduced) in the mixed modality blocks. To further highlight the latter result, we performed an additional analysis on half the data in each block only considering equivalent RT across single and mixed modality conditions (Q3-4 in the single modality blocks and Q2-3 in the mixed 
modality block). Within this subset of data the main finding was maintained. Namely, the Simon effect vanished in the mixed modality block but was significant in single modality blocks, according to a repeated measure ANOVA with Block (first single modality block, mixed modality and second single modality blocks) and $S-R$ compatibility (compatible/incompatible) as within subject variables. The interaction between Block and $S$ - $R$ compatibility was significant $\left[\mathrm{F}(2,28)=10.10, \mathrm{p}=0.0005, \eta^{2}=\right.$ 0.42]: the Simon effect was present in the single modality blocks (block 1: $t$ (14)=-3.68; $\mathrm{p}=0.002 ; \mathrm{d}_{\mathrm{z}}=0.97 ;$ block $\left.2: \mathrm{t}(14)=-3.76 ; \mathrm{p}=0.002 ; \mathrm{d}_{\mathrm{z}}=1.37\right)$ but not for the mixed modality block ( $\left.(14)=-0.657 ; p=0.522 ; d_{z}=0.25\right)$. In summary, although it is true that the Simon effect is modulated as function of overall RT, the effect of mixing modalities is present, even considering comparable RTs. The behavioral results set the grounds for the investigation of the neural correlates of the Simon effect in the single and mixed modality scenarios.
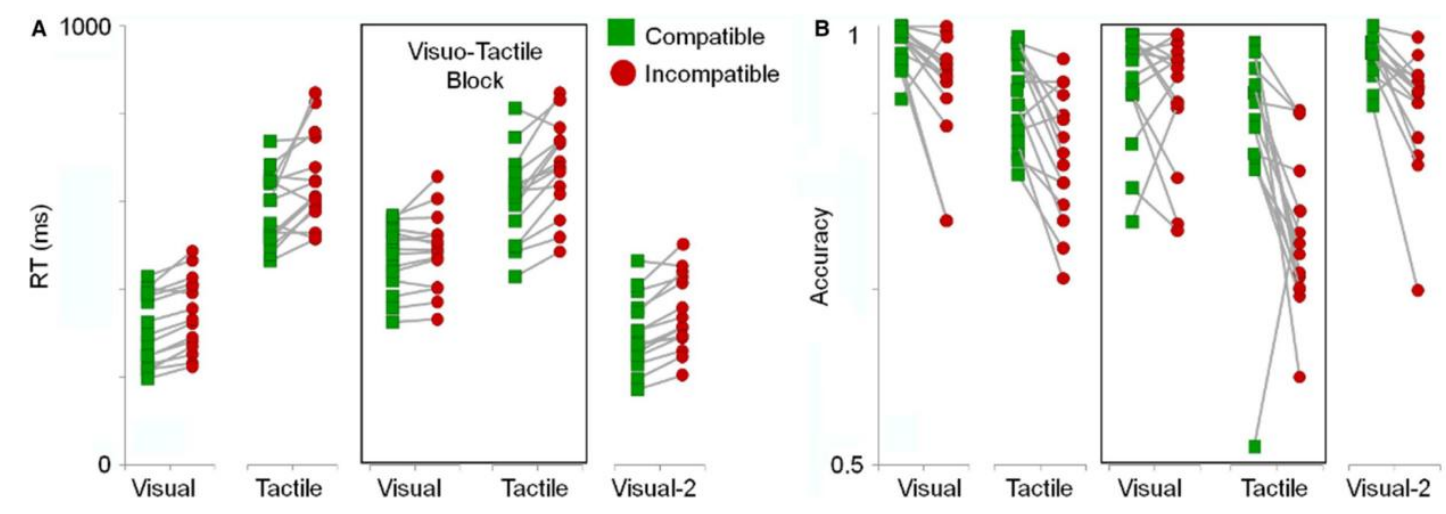

Figure 1. Behavioral results. RTs (A) and Accuracy (B) in the compatible (green) and incompatible (red) trials in the single modality (visual, tactile, visual-2) and mixed modality (visuo-tactile) blocks. Each point represents a participant $(\mathrm{N}=15)$.

\section{Event-related Potentials P300}

We first sought to find the P300 peak latency delay, typically found in incompatible Simon trials, in the single modality blocks of our study. Paired t-tests confirmed a P300 latency shift in the first single modality visual block (339 ms vs. $389 \mathrm{~ms}$ for compatible 
and incompatible trials; $\left.\mathrm{t}(14)=-4.39 ; \mathrm{p}<0.001 ; \mathrm{d}_{\mathrm{z}}=1.13\right)$ and the second single modality visual block ( 357 vs. $403 \mathrm{~ms} ; \mathrm{t}(14)=-3.99 ; \mathrm{p}=0.001 ; \mathrm{d}_{\mathrm{z}}=1.03$ ). We did not register a significant P300 latency shift between compatible and incompatible trials for the single modality tactile block (382 vs. $\left.389 \mathrm{~ms}, \mathrm{t}(14)=-0.74, \mathrm{p}=0.469 ; \mathrm{d}_{\mathrm{z}}=0.19\right)$.

The question of interest regarded the modulation of the P300 latency shift for visual trials in the mixed modality version of the Simon task. Therefore we ran a repeated measures ANOVA including data from single and mixed modality trial. Please note that just like for RT analyses, data from the second single visual modality block was not included here as it would render the design incomplete. This has been done for all the EEG indexes considered. The repetitive measures ANOVA included Context (single/mixed modality), Modality (visual/tactile), and S-R Compatibility (compatible/incompatible) as within participants' factors. Neither the effect of Context $\left[\mathrm{F}(1,14)=0.17, \mathrm{p}=0.685, \eta^{2}=0.01\right]$ nor Modality $\left[\mathrm{F}(1,14)=3.44, \mathrm{p}=0.085, \eta^{2}=0.20\right]$ resulted significant. The main effect of $S$ - $R$ Compatibility reached significance in the expected direction $($ compatible $=368 \mathrm{~ms}$ vs. incompatible $=384 \mathrm{~ms} ; \mathrm{F}(1,14)=4.97$, $\left.\mathrm{p}=0.043, \eta^{2}=0.26\right)$. Remarkably, the interaction between Context, Modality and $S-R$ Compatibility was significant $\left[\mathrm{F}(1,14)=7.80, \mathrm{p}=0.014, \eta^{2}=0.36\right]$. The interaction reflected the following pattern: in the visual trials there was a significant P300 latency shift in the single modality block (as already described above; compatible $=339 \mathrm{~ms}$ vs. incompatible $\left.=389 \mathrm{~ms} ; \mathrm{t}(14)=-4.39 ; \mathrm{p}<0.001 ; \mathrm{d}_{\mathrm{z}}=1.13\right)$ but not in the mixed modality block $\left(\right.$ compatible $=370 \mathrm{~ms}$ vs. incompatible $\left.=362 \mathrm{~ms} ; \mathrm{t}(14)=0.55, \mathrm{p}=0.592 ; \mathrm{d}_{\mathrm{z}}=0.14\right)$. For tactile trials, none of the task conditions revealed a P300 latency shift (single modality: compatible $=382 \mathrm{~ms}$ vs. incompatible $=389 \mathrm{~ms} ; \mathrm{t}(14)=-0.74, \mathrm{p}=0.469 ; \mathrm{d}_{\mathrm{z}}=0.19$; and in the mixed modality: compatible $=383 \mathrm{~ms}$ vs. incompatible=399 ms; $\mathrm{t}(14)=-1.39$, $\left.p=0.185 ; d_{z}=0.36\right)$. In general, the visual $P 300$ results are in line with the known 
literature (Ragot \& Renault, 1981; Valle-Inclán, 1996; Leuthold \& Sommer, 1999; Masaki et al., 2000; Ideno et al., 2005; Galashan et al., 2008; Melara et al., 2008). We recommend cautious interpretation regarding the failure to observe a P300 shift in the tactile modality given that, to the best of our knowledge, this is the first attempt to test P300 latency in a tactile Simon task.

Similarly as for the peak latency, we ran statistical comparisons on the P300 amplitude. None of the factors or interactions reached statistical significance in any of the blocks: as evident from Figure 2, P300 amplitude was very similar for compatible and incompatible trials in the single modality blocks $\left(\mathrm{t}_{\mathrm{visual}}(14)=-1.21, \mathrm{p}=0.247, \mathrm{~d}_{\mathrm{z}}=0.31\right.$; $\left.\mathrm{t}_{\text {visual2 }}(14)=-1.83, \mathrm{p}=0.09 ; \mathrm{d}_{\mathrm{z}}=0.47 ; \mathrm{t}_{\text {tactile }}(14)=0.19, \mathrm{p}=0.849 ; \mathrm{d}_{\mathrm{z}}=0.05\right)$, and in the visuotactile block $\left(\mathrm{t}_{\text {visual }}(14)=0.10, \mathrm{p}=0.920 ; \mathrm{d}_{\mathrm{z}}=0.03 ; \mathrm{t}_{\text {tactile }}(14)=0.77, \mathrm{p}=0.453 ; \mathrm{d}_{\mathrm{z}}=0.12\right)$. Collectively, the P300 results for the visual modality represent a similar pattern of that of the RT results. Specifically, P300 latency was modulated by compatibility (delayed P300 latency in incompatible trials in comparison with compatible trials) in the single modality visual blocks, while the effect disappeared in the visual trials of the mixed modality version of the task. For the tactile modality, however, the results were inconclusive, since no P300 latency effect was found in any of the conditions (single and mixed modality).

Since in the literature P300 latency and RTs have been found correlated (Ritter et al., 1972), we also performed a correlation analysis between P300 latency effect (incompatible - compatible) and Simon effect (RTs incompatible - compatible), separately for each block. However, we did not find any significant correlations (all pvalues $>0.27$ ) across participants. 

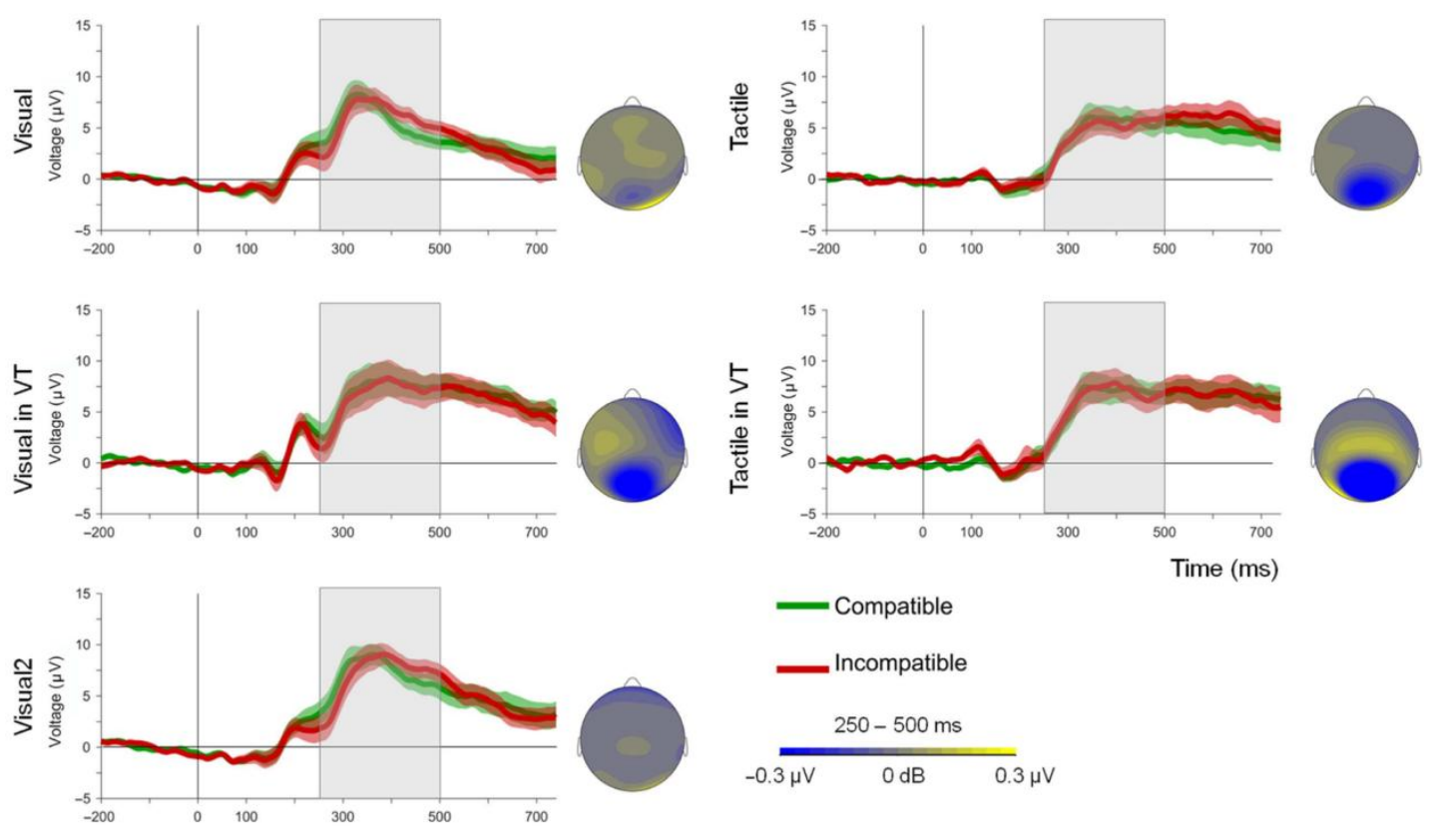

Figure 2. P300 waveforms for compatible (green line) and incompatible (red line) trials, for each block. Topographical distributions represent the difference in voltage between incompatible and compatible trials in the time window of interest $(250-500 \mathrm{~ms}$; shaded gray area) for each block. Error bars $=$ standard error of the mean (SEM).

\section{Theta power}

As explained in the methods section we extracted the average value (80ms) around the peaks in the theta power for each participant and condition, separately. First, we checked the differences between compatible and incompatible trials within single modality blocks, with the initial aim of replicating the previous finding for which theta power in incompatible trials is higher than in compatible trials, at least for vision (Nigbur et al., 2011; Cohen \& Donner, 2013; Cohen \& Ridderinkhof, 2013; Gulbinaite et al., 2014; Töllner et al., 2017).

In order to evaluate the theta power effect across blocks and modalities, we conducted a repeated measures ANOVA with data from single and mixed modality trials with Context (single/mixed modality), and Modality (visual/tactile) as within participants' factors, on the difference in theta power between incompatible and compatible trials. We found a main effect of Context, as theta power difference in the mixed modality 
block was lower than in the single modality blocks, regardless of modality

$\left[\mathrm{F}(1,13)=10.74, \mathrm{p}=0.007, \eta^{2}=0.47\right]$. The main effect of Modality was not significant

$\left[\mathrm{F}(1,13)=1.46, \mathrm{p}=0.250, \eta^{2}=0.11\right]$; nor the interaction between Context and Modality

$\left[F(1,12)=1.22, p=0.290, \eta^{2}=0.09\right]$. In accordance to the ANOVA results, we confirmed that in the mixed modality block, theta power was not different between incompatible and compatible trials for the visual $(10.90 \mathrm{~dB}$ vs. $11.05 \mathrm{~dB}, \mathrm{t}(13)=-1.06, \mathrm{p}=0.309$; $\left.\mathrm{d}_{\mathrm{z}}=0.28\right)$, nor for the tactile modality $(10.61 \mathrm{~dB}$ vs. $10.38 \mathrm{~dB}, \mathrm{t}(13)=0.95, \mathrm{p}=0.359$; $\left.d_{z}=0.25\right)$. Instead, as expected, in the single-modality visual Simon task, theta power in incompatible trials was higher than in compatible trials (10.47 vs. $10.97 \mathrm{~dB}$ respectively, $\left.\mathrm{t}(14)=-2.65, \mathrm{p}=0.019 ; \mathrm{d}_{\mathrm{z}}=0.68\right)$. Theta modulation was also found for the single modality tactile Simon task, in the expected direction (10.15 dB vs $10.71 \mathrm{~dB}$, $\left.t(13)=-6.89, p<0.001 ; d_{z}=1.84\right)$ (see Figure 3$)$. For completeness, we checked the contrast compatible vs. incompatible for the second single modality visual Simon block, which resulted in significant differences in the same direction as the initial single modality visual block $\left(10.83 \mathrm{~dB}\right.$ vs. $\left.11.31 \mathrm{~dB}, \mathrm{t}(13)=-2.91, \mathrm{p}=0.012 ; \mathrm{d}_{\mathrm{z}}=0.78\right)$.
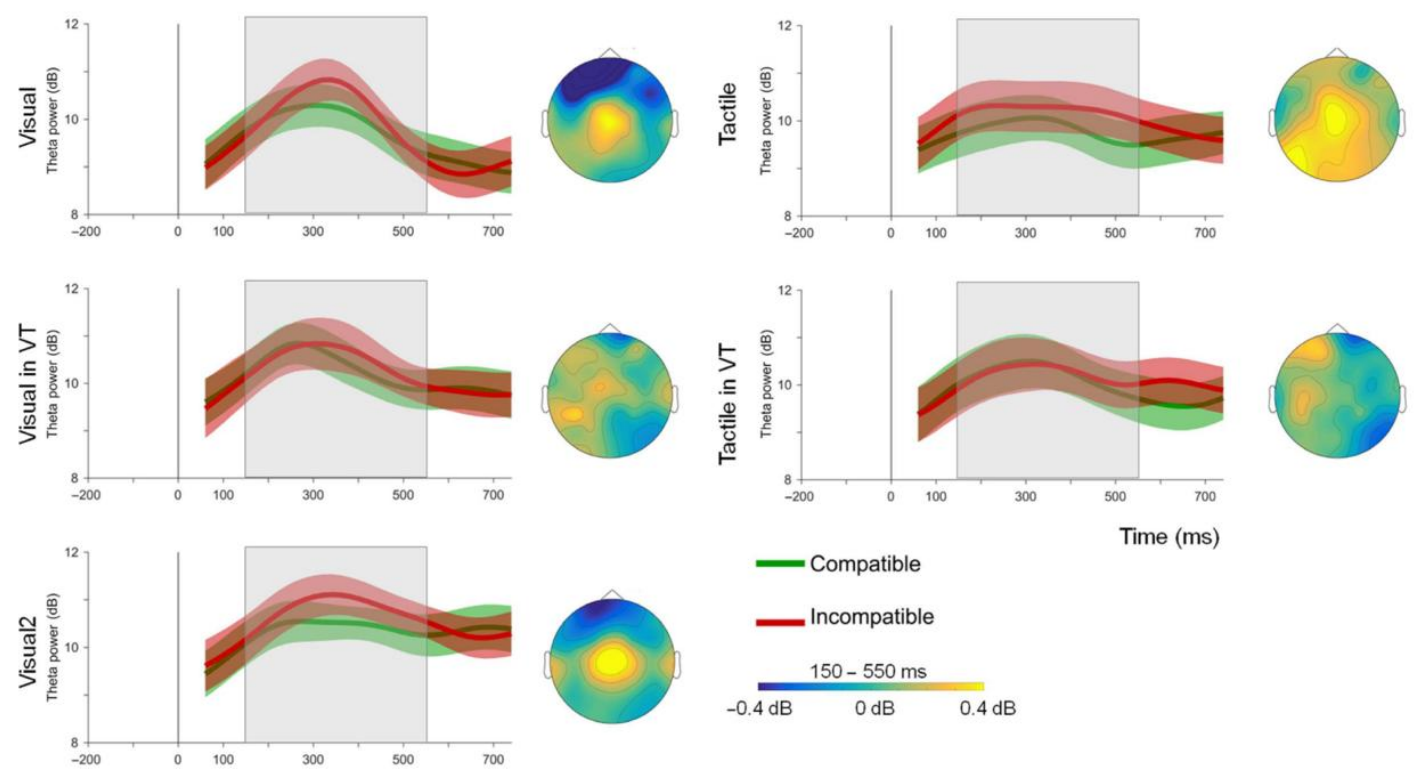

Figure 3. EEG power in the theta band $(5-7 \mathrm{~Hz})$ for compatible (green line) and incompatible (red line) trials. Topographical distributions the difference in theta power between incompatible and compatible trials in the time window of interest $(150-550 \mathrm{~ms}$; shaded gray area) for each block. Error bars $=$ standard 
error of the mean (SEM).

\section{Lateralized Readiness Potential}

We used a Surface Laplacian transformation of the EEG signal, in order to improve spatial resolution and prevent possible contamination of the motor potentials by sensory potentials due to the lateralized targets (Valle-Inclán, 1996; Eimer, 1998).

Consequently, the LRP amplitude values are expressed in $\mu \mathrm{V} / \mathrm{cm}^{3}$. As a first step in the analysis, we checked the absolute amplitude of the compatible and incompatible LRPs in order to ensure that a motor activation happened beyond spontaneous noise fluctuations (Vallesi et al., 2005). To do this, we compared the amplitude of the poststimulus LRP in $2 \mathrm{~ms}$ steps (corresponding to a sample) to the mean amplitude of 200 ms pre-stimulus (baseline) through paired t-tests across participants. Guthrie \& Buchwald's (1991) correction was applied to estimate significant deviations from baseline ( $\mathrm{p}<0.05$ for 15 consecutive sampling points, corresponding to $30 \mathrm{~ms}$ or more). The results from this analysis are graphically reported in Figure 4. This analysis showed that, in both the first and second single modality visual blocks, we found the typical LRP-dip, confirming prior results regarding the automatic motor activation of the response hand corresponding to the irrelevant stimulus location. However, in the mixed modality block the LRP-dip was not statistically different from baseline in visual trials (see Figure 4 and table 1). This means that, in contrast to the single modality blocks for which the irrelevant stimulus location triggers an activation of the motor pathway corresponding to a response toward the source of stimulation, in the mixed modality block the influence of stimulus location was negligible.

A second part of the LRP analysis we addressed is the relative differences between single and mixed modality trials. We focused on modulations of the LRP-dip peak amplitude (the positive deflection in incompatible trials against the negative deflection 
in compatible trials) across blocks. We extracted the LRP peak amplitude in the 80-200 ms window (stimulus-locked), based on the notion that the LRP-dip, if present, appears in an early time window (Vallesi et al., 2005). Please note that locking the LRP analysis to stimulus leads to temporal smoothing of the signal at longer latencies due to response time variability, rendering later components difficult to interpret. We then ran a repeated measures ANOVA on the LRP peak amplitude with Context (single/mixed modality), Modality (visual/tactile), and $S$-R Compatibility (compatible/incompatible) as within participants' factors. Only the effect of $S$ - $R$ Compatibility reached significance, because, as expected, incompatible trials had positive amplitudes compared to negative amplitudes in compatible trials (compatible $=-0.00040 \mu \mathrm{V} / \mathrm{cm}^{3} \mathrm{vs}$. incompatible $\left.=0.00045 \mu \mathrm{V} / \mathrm{cm}^{3} ; \mathrm{F}(1,13)=76.10, \mathrm{p}<0.001, \eta^{2}=0.85\right)$, and this pattern was present for all the conditions. The LRP peak amplitude corresponding to compatible and incompatible trials did not seem to differ among blocks and stimulus modality (Context x Modality x S-R Compatibility $\left[\mathrm{F}(1,13)=0.06 ; \mathrm{p}=0.806 ; \eta^{2}=0.00\right]$ ). Indeed, as showed in Figure 4, the LRPs trend is similar across conditions, including in the mixed modality visual trials, for which the contrast between compatible and incompatible LRP amplitudes is present (non significant ANOVA interaction), but reduced (non significant t-test against baseline). This could indicate a remnant of spatial processing in the mixed modality trials. However, only considering the visual modality blocks in a repetitive measures ANOVA with Blocks (visual single modality, visual trials in mixed modality, second visual single modality) and $S-R$ compatibility (compatible/incompatible) as factors, we found the expected significant interaction $(\mathrm{F}(2$, 26) $\left.=5.580 ; p=0.042 ; \eta^{2}=0.22\right)$. Collectively, we can conclude that the LRP results showed that the covert motor activation of the incorrect hand in the mixed modality block was strongly reduced (i.e., no difference compared to the baseline) with respect to 
the visual single modality blocks. In the tactile modality, we found the typical LRP-dip,

hence extending the finding of visual S-R compatibility tasks to the tactile modality.

Moreover, the tactile effect was not affected by mixing modalities, in accordance with

RTs evidence of the tactile Simon effect.
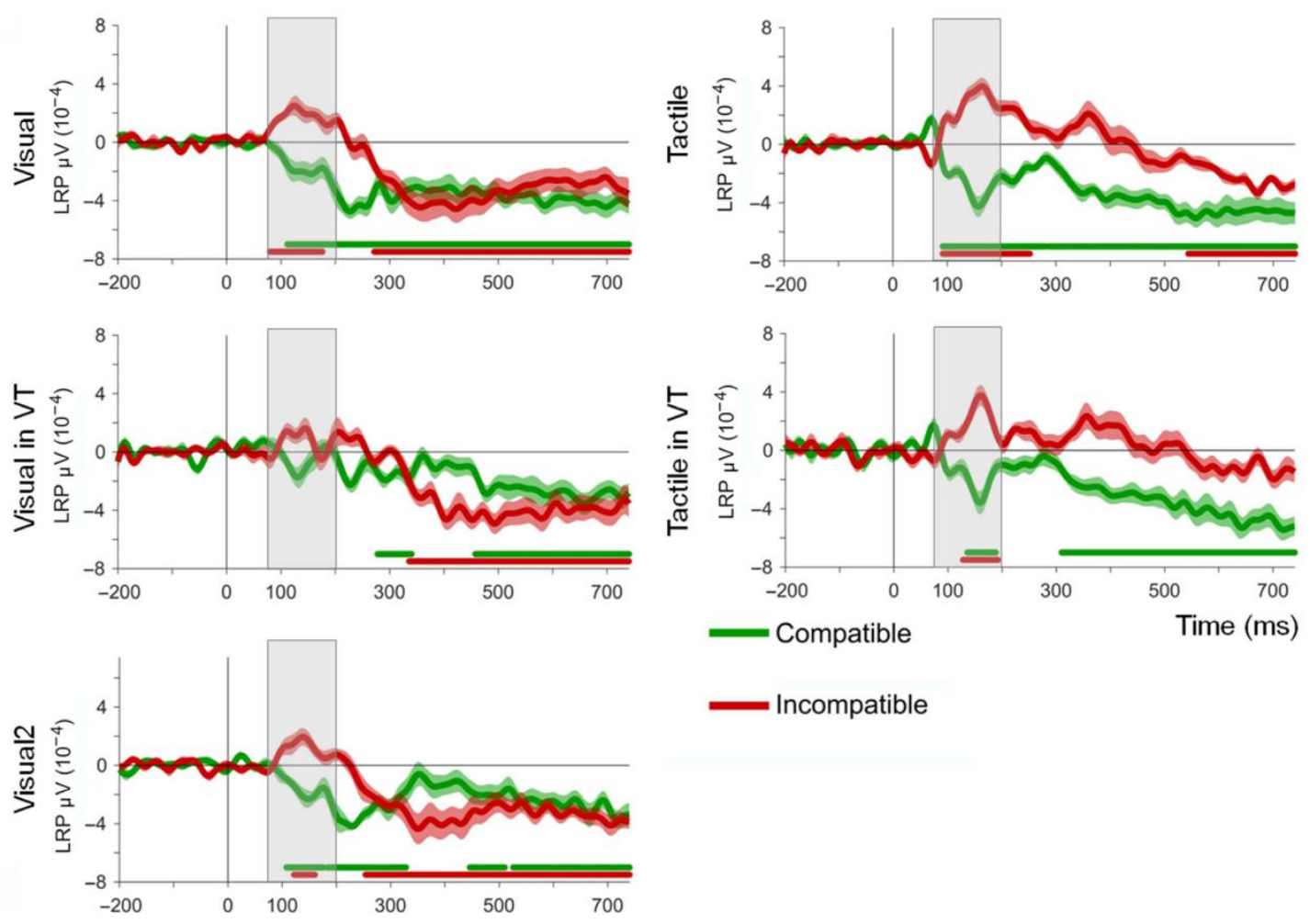

Incompatible

Figure 4. LRP waveforms for compatible (green line) and incompatible (red line) trials, for each block. Note that because we applied a Surface Laplacian transformation the LRP amplitude values are in $\mu \mathrm{V} / \mathrm{cm}^{4}$. Marked lines at the bottom of each graph (green and red for compatible and incompatible trials, respectively) represent the intervals at which the LRP wave was significantly different from baseline after Guthrie \& Buchwald's (1991) correction. The shaded gray area represents the window of interest (80-200 $\mathrm{ms}$ ) for the LRP-dip peak; Error bars= standard error of the mean (SEM).

\begin{tabular}{|c|c|c|c|c|c|c|c|c|c|c|}
\hline \multirow[t]{2}{*}{ Experimental block } & \multicolumn{2}{|c|}{$\begin{array}{l}\text { Visual single } \\
\text { modality block }\end{array}$} & \multicolumn{2}{|c|}{$\begin{array}{l}\text { Tactile single } \\
\text { modality block }\end{array}$} & \multicolumn{2}{|c|}{$\begin{array}{l}\text { Visual modality in the } \\
\text { mixed block }\end{array}$} & \multicolumn{2}{|c|}{$\begin{array}{l}\text { Tactile modality in } \\
\text { the mixed block }\end{array}$} & \multicolumn{2}{|c|}{$\begin{array}{l}\text { Visual single } \\
\text { modality block }\end{array}$} \\
\hline & Comp & Incomp & Comp & Incomp & Comp & Incomp & Comp & Incomp & Comp & Incomp \\
\hline LRP-dip & $\begin{array}{l}112-740 \\
(314 \mathrm{stp})\end{array}$ & $\begin{array}{l}80-176 \\
\text { (48 stp) }\end{array}$ & $\begin{array}{l}92-282 \\
(245 \mathrm{stp})\end{array}$ & $\begin{array}{l}92-252 \\
(80 \text { stp) }\end{array}$ & & & $\begin{array}{l}136-188 \\
(26 \mathrm{stp})\end{array}$ & $\begin{array}{r}128-192 \\
(32 \mathrm{stp})\end{array}$ & $\begin{array}{l}108-176 \\
(34 \mathrm{stp})\end{array}$ & $\begin{array}{l}122-160 \\
(19 \mathrm{stp})\end{array}$ \\
\hline $\begin{array}{l}\text { Correct hand } \\
\text { activation }\end{array}$ & & $\begin{array}{l}272-740 \\
(234 \mathrm{stp})\end{array}$ & $\begin{array}{l}288-740 \\
(226 \mathrm{stp})\end{array}$ & $\begin{array}{l}544-740 \\
(98 \mathrm{stp})\end{array}$ & $\begin{array}{l}278-340 \\
\text { (31 stp); } \\
458-740 \\
\text { (141 stp) }\end{array}$ & $\begin{array}{l}336-740 \\
(202 \mathrm{stp})\end{array}$ & $\begin{array}{l}310-740 \\
(215 \mathrm{stp})\end{array}$ & & $\begin{array}{l}184-328 \\
\text { (72 stp); } \\
446-510 \\
\text { (32 stp); } \\
526-740 \\
\text { (107 stp) }\end{array}$ & $\begin{array}{l}254-740 \\
(243 \mathrm{stp})\end{array}$ \\
\hline
\end{tabular}

Table 1. Intervals (ms) at which the LRP waves was significantly different from baseline after Guthrie \& Buchwald's (1991) correction for each of the Simon blocks, which correspond to the marked lines at the bottom of each graph in Figure 4. Stp $=$ significant time points $<\mathrm{p}$ value 0.005 (time point $=2 \mathrm{~ms}$ at a sample rate of $500 \mathrm{~Hz}$ ). 


\section{Discussion}

In this study, we turned to well-known neurophysiological indices previously associated to the Simon effect in vision. These markers have been interpreted as reflecting different but possibly interrelated aspects of the compatibility effect: perceptual interference (P300-latency shift), motor interference (LRP-dip) and cognitive conflict (theta power). The goal was to address the impact of irrelevant spatial information in the visual Simon effect under a mixed modality scenario. The interest of this study arises from previous results showing that the visual Simon effect falters in mixed modality scenario (Ruzzoli \& Soto-Faraco, 2017). Here, we replicated the disappearance of the visual Simon effect in mixed modality context, despite the effect persisted in the single modality contexts, as expected. At physiological level, we found no evidence for the processing of irrelevant spatial information from the visual event in the mixed modality context, in agreement with the behavioural pattern. In stark contrast, all expected EEG markers of the visual Simon effect were successfully found in the single modality blocks, consistent with the literature. Given the main interest in the visual modality, we will first focus on vision in the discussion of the results of each EEG marker, and then address a possible generalization to touch. Finally, we will discuss several implications of our findings in terms of spatial information processing.

\section{Visual EEG markers in the single vs. mixed modality scenarios}

The latency of P300 has often been associated with the duration of stimulus evaluation (Donchin \& Coles, 1988). According to this framework, Hasbroucq \& Guiard (1991) proposed that the Simon effect might arise from a stimulus-identification delay, which prolongs the time necessary for encoding the relevant stimulus dimension (i.e., the target colour) when its position is incompatible with the response goal. A longer stimulus-identification processing should delay P300 latency, which is indeed a robust 
indicator of the Simon effect (Ragot \& Renault, 1981; Leuthold, 2011). Our results are consistent with this literature, as we found the typical P300 peak latency delay in incompatible trials (Ragot \& Renault, 1981; Valle-Inclán, 1996; Leuthold \& Sommer, 1999; Masaki et al., 2000; Ideno et al., 2005; Galashan et al., 2008; Melara et al., 2008). However, when visual trials were intermixed with tactile trials, the P300 delay disappeared, as did the RT effect. This result suggests that in the mixed modality block, irrelevant spatial information from stimulus location, which usually gives rise to the SR compatibility effect, fails to exert an effect on the P300 latency (Ragot \& Renault, 1981; Valle-Inclán, 1996). Although some studies in the literature have reported that P300 peak latency correlates with RTs (Ritter et al., 1972), we did not find significant correlation between P300 latency shift (incompatible - compatible trials) and Simon effect across participants. This lack of correlation might arise from the relative small sample size $(\mathrm{N}=15)$ in this study, as well as to the fact that trial to trial correlations (as found in Ritter et al., 1972) might not necessarily reflect as participant to participant correlations.

The second neural marker we have considered in this study was the modulation in the power of the theta frequency band $(5-7 \mathrm{~Hz})$ at middle frontal electrodes typically considered to reflect cognitive conflict (Nigbur et al., 2011; Cavanagh et al., 2012; Cohen, 2014). The results of the present study indicated that, albeit theta power effects appeared in single modality blocks as expected, they were no longer present in the mixed modality scenarios for the visual (and tactile) modality. Hence, like in the case of P300, theta power modulation paralleled the vanishing of the Simon effect in RTs in the mixed modality block, indicating that spatial information coming from stimulus location did not seem to penetrate executive control and generate cognitive conflict 
between the irrelevant location of the stimulus and the location of the intended (correct) response.

Finally, we also investigated the impact of interference at the motor level (i.e., LRP-dip) since the Simon effect is thought to originate from automatic response priming (Lu \& Proctor, 1995). We reasoned that the LRP-dip might signal the influence of spatial information in the mixed modality version of the visual Simon effect, even if it no longer generates RT effects down the stream. In the present study, the single modality LRPs were in line with prior literature (Valle-Inclán, 1996; Vallesi et al., 2005; Cespón et al., 2012), with evidence of LRP-dip (incorrect hand activation in the incompatible trials). Yet, in the mixed modality block, the LRP-dip in the visual trials was reduced to an insignificant numerical trend, suggesting that, similarly to RTs, in the mixed modality scenario the impact of irrelevant spatial information was strongly reduced also at neurophysiological level.

\section{Extrapolation of Visual EEG markers to Tactile Simon Task}

It is important to note that EEG markers (the P300, the LRP and theta power modulations) in the Simon task have been mainly investigated by means of visual stimuli in the past (for P300: Ragot \& Renault, 1981; Valle-Inclán, 1996; Leuthold \& Sommer, 1999; Masaki et al., 2000; Ideno et al., 2005; Galashan et al., 2008; Melara et al., 2008a; for theta power: Nigbur et al., 2011a; Cohen \& Donner, 2013a; Cohen \& Ridderinkhof, 2013; Gulbinaite et al., 2014; Töllner et al., 2017; for LRP: Valle-Inclán, 1996; Vallesi et al., 2005; Cespón et al., 2012). To the best of our knowledge, no studies before have used tactile stimuli with the aim of generalizing the interpretation of the physiological mechanisms underlying the Simon effect. Therefore, in the context of this study, we could only start from the assumption that the P300 latency effect, the 
LRP-dip effect and the theta power modulation, widely described for the visual modality in the literature, would be similar for the tactile modality in the Simon task. This hypothesis, however, is based on the (naïve) assumption that physiological correlates of the tactile Simon task are similar to those in vision. In fact, according to our analyses of the EEG correlates for the tactile Simon task, this assumption did not hold true.

Regarding the P300 delay, when it comes to the tactile Simon task, the data did not show P300 peak latency effects neither in the single nor in the mixed modality blocks, therefore the results are inconclusive. Conversely, theta power modulation was shown in the tactile single modality Simon block. Although this effect is far from new in the visual modality (Cohen, 2014), we must note that it extends to the tactile modality too, a novel finding as far as we are aware. Yet, in the mixed modality context, the tactile theta power modulation was not present, despite the presence of the Simon effect in RTs. The interpretation of the lack of modulation of theta power in the tactile mixed modality scenario remains uncertain, much like the lack of P300 latency effects in touch altogether. This piece of evidence offers a possible neural correlate of the asymmetry between visual and tactile RTs, in the Simon effect in the mixed modality context, in which tactile Simon effect is present at the detriment of the visual Simon effect. The evidence regarding the LRP index in the tactile modality allows us to generalize from the visual to the tactile modality. It is perhaps interesting to note that unlike the P300 and the theta EEG markers, the pattern of the LRP effect (visual single modality block, and both single and mixed modality tactile blocks) was in accordance with the RT pattern for both modalities in the two tested scenarios. This pattern suggests that the LRP, reflecting congruency at response-selection stage (Lu \& Proctor, 1995), might be a modality-unspecific marker for the Simon effect. Even though, the EEG evidence for 
the Simon effect within the tactile modality was weaker than for the visual modality (see the P300 effect in the single modality block) the results presented here can provide a basis for the generalization of the physiological marker of the Simon effect to the tactile modality. This is an important step forward in the S-R compatibility literature which can shed new light on the design of human computer interfaces (Proctor \& Vu, 2016), which are mainly centred on the visual (or audio-visual) modality.

\section{The breakdown of the visual Simon effect in mixed modality scenarios: Theoretical implications}

In the single modality blocks, which served as reality checks, EEG and behavioral markers of the typical visual Simon effect were invariably observed as expected, thus suggesting that the experimental protocol was sensitive enough to highlight the impact of irrelevant spatial information in our setting. Remarkably, performance in the single modality visual blocks ran after and before the mixed modality block was equivalent, suggesting that the collapse (or at best, strong attenuation) of visual S-R compatibility was contingent to the mixed modality context, and short lived. Furthermore, we can also exclude fatigue as an explanation for the steep reduction of the visual Simon effect in the mixed modality context. We can therefore conclude that the modality context (i.e., uni- or multi-sensory) may determine the degree to which (irrelevant) spatial information is processed in vision. The question then is: What is the peculiarity of the mixed modality (visuo-tactile) Simon task?

As suggested in Ruzzoli and Soto-Faraco (2017), the unpredictable switching between spatial reference frames (external and anatomical) associated to each modality (visual and tactile, respectively) might determine the degree to which irrelevant spatial information is processed. Indeed, one influential theoretical framework assumes that the Simon effect (or spatial S-R compatibility) emerges according to a system of 
coordinates automatically used to locate events in space (i.e., reference frame) (Wallace, 1971; Hommel, 1993). In this context, it is interesting to highlight that when multiple reference frames are simultaneously at play in a given task, even within modality, some instances of S-R compatibility disappear (Ladavas \& Moscovitch, 1984; Umiltà \& Liotti, 1987; Stoffer, 1991; Lamberts et al., 1992; Hommel, 1993, 1994; Roswarski \& Proctor, 1996). Our results sit well within this framework, because reference frame varies according to the sensory modality of the stimulus, and hence, the consequence of the mixed modality context is that different reference frames are at play (Wallace, 1971; Medina et al., 2014; Ruzzoli \& Soto-Faraco, 2017). Indeed, when the Simon task involves switching between modalities which abide to the same reference frame, such as vision and audition, the Simon effect is maintained for both modalities (Ruzzoli \& SotoFaraco, 2017 experiment 5).

This explanation, however, still leaves one important aspect of our results unexplained. Why does irrelevant spatial information stop influencing visual trials under mixed modalities, but still produces the tactile Simon effect? What is more, such an asymmetry in favor of touch in the Simon task (Medina et al., 2014; Ruzzoli \& Soto-Faraco, 2017) appears to stand in contradiction with previous findings highlighting the extremely important role of a visually-based external reference frame for encoding and responding to tactile events on the body (Yamamoto \& Kitazawa, 2001; Röder et al., 2007; Azañón \& Soto-Faraco, 2008; Heed et al., 2015). Please note that, like in the present study, most of these previous studies on tactile remapping, in which the external-visual reference frame prevails, have occluded the hands of the participant in their protocols (Yamamoto \& Kitazawa, 2001; Röder et al., 2007; Azañón \& Soto-Faraco, 2008; Heed et al., 2015), thus excluding the alternative explanation that by occluding the hands 
from sight we could have obliterated the usual dominance of visually-based encoding of spatial information.

In order to understand this apparent contradiction, and to attempt to explain the asymmetry found between visual and tactile Simon effects, it is useful to consider the way the cognitive system represents spatial information in both kinds of tasks (i.e., the Simon task and the tactile remapping tasks used in previous studies highlighting the role of external reference frame for touch). We think that four important points deserve discussion regarding how spatial information is represented and transformed in the brain. We discuss them below.

\section{Spatial information is pivotal in the Simon and remapping tasks}

Notwithstanding space might or might not be considered task relevant: in the Simon task studies and in many previous studies about spatial remapping of touch (e.g., temporal order judgments, TOJ; and cross-modal cueing) some form of spatial encoding is pivotal to solve the task. In remapping studies, space is often relevant because the task goal is to respond to stimulus location, explicitly or implicitly (Yamamoto \& Kitazawa, 2001; Azañón \& Soto-Faraco, 2008; Heed et al., 2015). In fact, the influence of the external spatial reference frame in the tactile processing, what is commonly referred to as tactile remapping, tends to disappear when space is removed from any aspect of the task, including stimulus, S-R mapping and response (Gallace et al., 2008; Azañón et al., 2010). In the Simon effect, according to task instructions, space is irrelevant but is cardinal for the Simon effect to arise because, in order to respond, actions play out according to a motor spatial reference frame.

\section{The relevant reference frame is computed involuntarily but incurs costs}

In tasks where space is pivotal, such as the Simon task, the computation of a spatial reference frame seems to be a mandatory process which refers spatial information to a 
unique system of coordinates. However, computing such relevant reference frame involves weighting different sources of spatial information according to task demand and context (see also Kornblum et al., 1990; Pouget et al., 2002). Mixed modality scenarios involve referring information of an upcoming event under uncertainty about its sensory modality, spatial location, and task-relevant contents (colour, frequency). This situation is more demanding than when the sensory modality and task-relevant information are known in advance (single modality case).

\section{The relevant reference frame is computed flexibly}

In situations in which space is pivotal to resolve the task (first point above) the involuntary computation of the relevant spatial reference frame (second point above) might be a matter of task context and demands, rather than a hardwired (modalitydependent) process. This view stands in contrast with the often held assumption that the external (visual) reference frame is dominant over the anatomical (tactile) reference frame (Yamamoto \& Kitazawa, 2001; Azañón \& Soto-Faraco, 2008; Medina et al., 2014; Heed et al., 2015). We believe that the resilience of an anatomical reference frame over the external visual one in the mixed modality Simon task might be the consequence of the weight each reference frame is given in this particular context, instead of a hardwired parameter. That is, processing of irrelevant spatial information (i.e., what is measured by the Simon task) might be rather flexible, and it might be a consequence of how the system is set up given available task relevant information, task goal and available remaining-resources.

\section{Only one relevant reference frame can be computed and used at a time}

Above, we have highlighted that spatial information is pivotal in the Simon task and in most tasks addressing tactile remapping. Also, in such situations, a reference frame will be computed involuntarily but flexibly, depending on task relevant aspects and context. 
A fourth point in our proposal is that that the emergence of a reference frame is the outcome of a weighting process that has only one output. Potentially, there can be many possible different reference frames, but at any one time only one takes effective control. Accordingly, here, we found that spatial information from the visual event did not have an impact (in RTs or neural markers) when more than one reference frame is possible to be computed.

Such conclusion can be controversial according to the attentional hypothesis for the Simon effect (Nicoletti \& Umiltà, 1994) or the previously mentioned tactile remapping literature (Yamamoto \& Kitazawa, 2001; Azañón \& Soto-Faraco, 2008; Heed et al., 2015). Specifically, the attentional account states that multiple reference frames can be used based on the focus of attention (left/right with respect to the body midline, left/right with respect to the left/right position of a fixation cross) (Lamberts et al., 1992). Tactile remapping studies, instead, propose that in order to react to a stimulus on the body, spatial information derived from stimulus location is automatically transformed from an anatomical reference frame into an external one (Yamamoto \& Kitazawa, 2001; Azañón \& Soto-Faraco, 2008; Heed et al., 2015). Both these perspectives suggest that various reference frames are computed and that they can influence one another (Medina et al., 2014). We propose that a reference frame is the output of a computation (not under voluntary control) which already takes into account the different sources of spatial information present in the actual context (Pouget et al., 2002).

In the mixed modality scenarios, or other situations in which multiple competing spatial reference frames are possible (Ladavas \& Moscovitch, 1984; Umiltà \& Liotti, 1987; Stoffer, 1991; Lamberts et al., 1992; Hommel, 1993; Roswarski \& Proctor, 1996; Hommel et al., 2001), the degree of processing of irrelevant spatial information may 
remain more superficial (hence, not allowing resources for remapping). The fact that touch is harder to shut down under these high-load circumstances (see also Berlucchi et $a l .$, 1977), is probably because touch triggers an egocentric reference frame where stimulus location and response location relates to body posture (tactile sensation vs. proprioception). Finally, the transformations needed to go from stimulus to response might be more resource consuming and hence lead to lesser processing of the (irrelevant) spatial location in the Simon task, compared to TOJ tasks like the one used in Yamamoto \& Kitazawa (2001) and other related studies (Azañón \& Soto-Faraco, 2008; Heed et al., 2015).

\section{Conclusion}

By means of a mixed modality Simon task in an EEG protocol we have showed that irrelevant spatial information in vision, which classically exerts a strong effect on responses, fails to affect performance at the behavioral level as well as to produce neurophysiological evidence of spatial interference. This break down of spatial interference did not occur for touch. The present results are surprising, given that, spatial congruency effects appear reliably in a wide variety of contexts and, visualbased spatial reference frame often prevails over the tactile-based anatomical one. To explain this apparent anomaly we suggest that in a task in which a spatial representation is pivotal, the relevant reference frame is built up involuntarily (as previous hypotheses suggest) but rather more flexibly than previously thought, as a function of the context and task demands. Our conclusion opens up to possible experimental tests. For example, one would expect that in the mixed modality context, it would be possible to observe the visual Simon effect if the upcoming modality would be cued in advance, thereby reducing uncertainty regarding the relevant frame of reference for the upcoming stimulus. In this situation, given the appropriate time for computing-the relevant reference frame, we expect participants to be able to effectively switch between spatial reference frames (external, anatomical) from trial to trial, thereby being prone to the 
influence of the irrelevant stimulus location. This result can also have an impact on human-computer interface design; for example, we can expect that the influence of alerting vibrations on the body from some smart devices integrated in the body schema (e.g., cell-phone, watch) might induce involuntary switches toward an anatomical reference frame that can be potentially detrimental for tasks requiring visual spatial attention, such as driving (longer RTs, less influence of spatial information). 


\section{Bibliography}

Azañón, E., Camacho, K., \& Soto-Faraco, S. (2010) Tactile remapping beyond space. Eur. J. Neurosci., 31, 1858-1867.

Azañón, E. \& Soto-Faraco, S. (2008) Changing Reference Frames during the Encoding of Tactile Events. Curr. Biol., 18, 1044-1049.

Başar, E., Başar-Eroğlu, C., Karakaş, S., \& Schürmann, M. (1999) Are cognitive processes manifested in event-related gamma, alpha, theta and delta oscillations in the EEG? Neurosci. Lett., 259, 165-168.

Başar, E., Başar-Eroglu, C., Parnefjord, R., Rahn, E., \& Schürmann, M. (1992) Evoked Potentials: Ensembles of Brain Induced Rhythmicities in the Alpha, Theta and Gamma Ranges. In Induced Rhythms in the Brain. Birkhäuser Boston, Boston, MA, pp. 155-181.

Berlucchi, G., Crea, F., Di Stefano, M., \& Tassinari, G. (1977) Influence of spatial stimulus-response compatibility on reaction time of ipsilateral and contralateral hand to lateralized light stimuli. J. Exp. Psychol. Hum. Percept. Perform., 3, 505517.

Brainard, D.H. (1997) The Psychophysics Toolbox. Spat. Vis., 10, 433-436.

Carvalhaes, C. \& de Barros, J.A. (2015) The surface Laplacian technique in EEG: Theory and methods. Int. J. Psychophysiol., 97, 174-188.

Cavanagh, J.F., Zambrano-Vazquez, L., \& Allen, J.J.B. (2012) Theta lingua franca: A common mid-frontal substrate for action monitoring processes. Psychophysiology, 49, 220-238.

Cespón, J., Galdo-Álvarez, S., \& Díaz, F. (2012) The Simon effect modulates N2cc and LRP but not the N2pc component. Int. J. Psychophysiol., 84, 120-129.

Cohen, M.X. (2014) A neural microcircuit for cognitive conflict detection and 
signaling. Trends Neurosci., 37, 480-490.

Cohen, M.X. \& Donner, T.H. (2013) Midfrontal conflict-related theta-band power reflects neural oscillations that predict behavior. J. Neurophysiol., 110, 2752-2763.

Cohen, M.X. \& Ridderinkhof, K.R. (2013) EEG Source Reconstruction Reveals Frontal-Parietal Dynamics of Spatial Conflict Processing. PLoS One, 8, e57293.

Coles, M.G. (1989) Modern mind-brain reading: psychophysiology, physiology, and cognition. Psychophysiology, 26, 251-269.

De Jong, R., Liang, C.-C., \& Lauber, E. (1994) Conditional and Unconditional Automaticity: A Dual-Process Model of Effects of Spatial Stimulus-Response Correspondence. J. Exp. Psychol. Hum. Percept. Perform., 20, 731-750.

Donchin, E. \& Coles, M.G. (1988) Is the P300 component a manifestation of context updating? Behav. Brain Sci., 11, 357-427.

Eimer, M. (1998) The lateralized readiness potential as an on-line measure of central response activation processes. Behav. Res. Methods, Instruments, Comput., 30, $146-156$.

Eriksen, B.A. \& Eriksen, C.W. (1974) Effects of noise letters upon the identification of a target letter in a nonsearch task. Percept. Psychophys., 16, 143-149.

Galashan, D., Wittfoth, M., Fehr, T., \& Herrmann, M. (2008) Two Simon tasks with different sources of conflict: An ERP study of motion- and location-based compatibility effects. Biol. Psychol., 78, 246-252.

Gallace, A., Soto-Faraco, S., Dalton, P., Kreukniet, B., \& Spence, C. (2008) Response requirements modulate tactile spatial congruency effects. Exp. Brain Res., 191, $171-186$.

Gratton, G., Coles, M.G., Sirevaag, E.J., Eriksen, C.W., \& Donchin, E. (1988) Pre- and poststimulus activation of response channels: A psychophysiological analysis. $J$. 
Exp. Psychol. Hum. Percept. Perform., 14, 331-344.

Gulbinaite, R., van Rijn, H., \& Cohen, M.X. (2014) Fronto-parietal network oscillations reveal relationship between working memory capacity and cognitive control. Front. Hum. Neurosci., 8, 761.

Guthrie, D. \& Buchwald, J.S. (1991) Significance testing of difference potentials. Psychophysiology, 28, 240-244.

Hasbroucq, T. \& Guiard, Y. (1991) Stimulus-response compatibility and the Simon effect: toward a conceptual clarification. J. Exp. Psychol. Hum. Percept. Perform., 17, 246-266.

Heed, T., Buchholz, V.N., Engel, A.K., \& Röder, B. (2015) Tactile remapping: From coordinate transformation to integration in sensorimotor processing. Trends Cogn. Sci., 19, 251-258.

Hommel (2011) The Simon effect as tool and heuristic. Acta Psychol. (Amst)., 136, 189-202.

Hommel, B. (1993) The role of attention for the Simon effect. Psychol. Res., 55, 208222.

Hommel, B. (1994) Effects of irrelevant spatial S-R compatibility depend on stimulus complexity. Psychol. Res., 56, 179-184.

Hommel, B., Müsseler, J., Aschersleben, G., \& Prinz, W. (2001) The Theory of Event Coding (TEC): a framework for perception and action planning. Behav. Brain Sci., 24, 849-78-937.

Ideno, T., Ishizu, T., Tujii, T., \& Kojima, S. (2005) Event-related potentials in the Simon task. Int. Congr. Ser., 1278, 131-134.

Kayser, J. \& Tenke, C.E. (2015) On the benefits of using surface Laplacian (current source density) methodology in electrophysiology. 
Kornblum, S., Hasbroucq, T., \& Osman, a (1990) Dimensional overlap: cognitive basis for stimulus-response compatibility--a model and taxonomy. Psychol. Rev., 97, $253-270$.

Ladavas, E. \& Moscovitch, M. (1984) Must egocentric and environmental frames of reference be aligned to produce spatial S-R compatibility effects? J. Exp. Psychol. Hum. Percept. Perform., 10, 205-215.

Lamberts, K., Tavernier, G., \& d'Ydewalle, G. (1992) Effects of multiple reference points in spatial stimulus-response compatibility. Acta Psychol. (Amst)., 79, 115130.

Leuthold, H. (2011) The Simon effect in cognitive electrophysiology: A short review. Acta Psychol. (Amst)., 136, 203-211.

Leuthold, H. \& Sommer, W. (1999) ERP correlates of error processing in spatial S-R compatibility tasks. Clin. Neurophysiol., 110, 342-357.

Lu, C.-H. \& Proctor, R.W. (1995) The influence of irrelevant location information on performance: A review of the Simon and spatial Stroop effects. Psychon. Bull. Rev., 2, 174-207.

Masaki, H., Takasawa, N., \& Yamazaki, K. (2000) An electrophysiological study of the locus of the interference effect in a stimulus-response compatibility paradigm. Psychophysiology, 37, 464-472.

Medina, J., McCloskey, M., Coslett, H.B., \& Rapp, B. (2014) Somatotopic representation of location: Evidence from the Simon effect. J. Exp. Psychol. Hum. Percept. Perform., 40, 2131-2142.

Melara, R.D., Wang, H., Vu, K.-P.L., \& Proctor, R.W. (2008) Attentional origins of the Simon effect: behavioral and electrophysiological evidence. Brain Res., 1215, $147-159$. 
Nicoletti, R. \& Umiltà, C. (1994) Attention shifts produce spatial stimulus codes. Psychol. Res., 56, 144-150.

Nigbur, R., Ivanova, G., \& Stürmer, B. (2011) Theta power as a marker for cognitive interference. Clin. Neurophysiol., 122, 2185-2194.

Oostenveld, R., Fries, P., Maris, E., \& Schoffelen, J.-M. (2011) FieldTrip: Open source software for advanced analysis of MEG, EEG, and invasive electrophysiological data. Comput. Intell. Neurosci., 2011, 156869.

Perrin, F., Pernier, J., Bertrand, O., \& Echallier, J.F. (1989) Spherical splines for scalp potential and current density mapping. Electroencephalogr. Clin. Neurophysiol., 72, 184-187.

Pouget, A., Deneve, S., \& Duhamel, J.-R. (2002) A computational perspective on the neural basis of multisensory spatial representations. Nat. Rev. Neurosci., 3, 741747.

Proctor, R.W. \& Lu, C.H. (1999) Processing irrelevant location information: practice and transfer effects in choice-reaction tasks. Mem. Cognit., 27, 63-77.

Proctor, R.W. \& Vu, K.-P.L. (2016) Principles for Designing Interfaces Compatible With Human Information Processing. Int. J. Hum. Comput. Interact., 32, 2-22.

Ragot, R. \& Renault, B. (1981) P300, as a function of S-R compatibility and motor programming. Biol. Psychol., 13, 289-294.

Riggio, L., Gawryszewski, L. de G., \& Umiltà, C. (1986) What is crossed in crossedhand effects? Acta Psychol. (Amst)., 62, 89-100.

Ritter, W., Simson, R., \& Vaughan, H.G. (1972) Association cortex potentials and reaction time in auditory discrimination. Electroencephalogr. Clin. Neurophysiol., $33,547-555$.

Röder, B., Kusmierek, A., Spence, C., \& Schicke, T. (2007) Developmental vision 
determines the reference frame for the multisensory control of action. Proc. Natl. Acad. Sci. U. S. A., 104, 4753-4758.

Roswarski, T.E. \& Proctor, R.W. (1996) Multiple spatial codes and temporal overlap in choice-reaction tasks. Psychol. Res., 59, 196-211.

Ruzzoli, M. \& Soto-Faraco, S. (2017) Modality-Switching in the Simon Task: The Clash of Reference Frames. J. Exp. Psychol. Gen., 146, 1478-1497.

Shenhav, A., Botvinick, M., \& Cohen, J. (2013) The expected value of control: An integrative theory of anterior cingulate cortex function. Neuron, 79, 217-240.

Simon, J.R., Hinrichs, J. V, \& Craft, J.L. (1970) Auditory S-R compatibility: reaction time as a function of ear-hand correspondence and ear-response-location correspondence. J. Exp. Psychol., 86, 97-102.

Simon, J.R. \& Small, A.M. (1969) Processing auditory information: interference from an irrelevant cue. J. Appl. Psychol., 53, 433-435.

Stoffer, T.H. (1991) Attentional focussing and spatial stimulus-response compatibility. Psychol. Res., 53, 127-135.

Stroop, J.R. (1935) Studies of interference in serial verbal reactions. J. Exp. Psychol., 18, 643-662.

Töllner, T., Wang, Y., Makeig, S., Müller, H.J., Jung, T.-P., \& Gramann, K. (2017) Two Independent Frontal Midline Theta Oscillations during Conflict Detection and Adaptation in a Simon-Type Manual Reaching Task. J. Neurosci., 37, 2504-2515.

Umiltà, C. \& Liotti, M. (1987) Egocentric and relative spatial codes in S-R compatibility. Psychol. Res., 49, 81-90.

Valle-Inclán, F. (1996) The locus of interference in the Simon effect: an ERP study. Biol. Psychol., 43, 147-162.

Vallesi, A., Mapelli, D., Schiff, S., Amodio, P., \& Umiltà, C. (2005) Horizontal and 
vertical Simon effect: different underlying mechanisms? Cognition, 96, B33-43.

Wallace, R.J. (1971) S-R compatibility and the idea of a response code. J. Exp. Psychol., 88, 354-360.

Wang, H., Liu, N., Zou, G., Li, H., Zeng, H., Chen, J., \& Chen, Q. (2016) The Simon effect based on the egocentric and allocentric reference frame. Attention, Perception, Psychophys., 78, 427-436.

Yamamoto, S. \& Kitazawa, S. (2001) Reversal of subjective temporal order due to arm crossing. Nat. Neurosci., 4, 759-765. 


\section{Acknowledgement:}

This research was supported by the Ministerio de Economia, Industria y Competividad (AEI/FEDER-PSI2016-75558-P to SSF; and Explora Ciencia 2015 PSI2015-72568EXP-AEI to MR), and AGAUR Generalitat de Catalunya (2014SGR856 to SSF).

\section{Conflict of interest:}

The authors have no conflict-of-interest to declare.

\section{Data sharing statement}

Data are still under exploitation for other hypothesis testing different than the ones presented in this study. Consequently, data will not be made public immediately after the publication of this manuscript. 